\title{
Nondestructive characterization of flake graphite cast iron by
}

\section{Magnetic Adaptive Testing}

\author{
Gábor Vértesy ${ }^{1}$, Tetsuya Uchimoto ${ }^{2}$, Toshiyuki Takagi ${ }^{2}$, Ivan Tomáğ and Hidehiko \\ Kage $^{4}$ \\ ${ }^{1}$ Hungarian Academy of Sciences, Institute of Technical Physics and Materials Science, \\ Budapest, Hungary \\ ${ }^{2}$ Institute of Fluid Science, Tohoku University, Sendai, Japan \\ ${ }^{3}$ Center of Advanced and Innovative Technologies, VSB-TUO, Ostrava, Czech \\ Republic \\ ${ }^{4}$ Kusaka Rare Metal Products Co., Tokyo, Japan
}

\begin{abstract}
Three series of flake graphite cast iron samples having different chemical compositions and different heat treatments within each series were investigated by the method of Magnetic Adaptive Testing. The flat samples were magnetized by an attached yoke, and sensitive descriptors were obtained from the proper evaluation, based on the measurements of series of magnetic minor hysteresis loops, without magnetic saturation of the samples. Results of the non-destructive magnetic tests were compared with the destructive mechanical measurements of Brinell hardness and linear correlation was found between them in all cases, where the influence of chemical composition and influence of heat treatment were considered.
\end{abstract}


Keywords: Cast iron, Magnetic hysteresis, Magnetic adaptive testing, Nondestructive testing

\section{Introduction}

Cast iron is one of the most frequently used industrial construction materials. Low cost of production, good machinability, and excellent possibilities of shaping the details by casting attract an intense interest of industry. The cast irons are generally manycomponent alloys of iron with large content of carbon. The cast iron structure is classified by its metallic matrix composition (ferrite, pearlite, carbides, etc.) and by morphology of its graphite inclusion. The mechanical properties are fundamentally dependent both on the matrix composition and on the graphite shape (flaky, spheroidal, vermicular, etc.), size and density [1]. One of the types of cast iron - the flake graphite cast iron - is frequently used for mechanical components in bearings, brake shoes, etc. because of its high wear resistance and damping capacity. The flake graphite cast iron is an ideal material for automobile brake disks since it has excellent damping properties and thermal conductivity just because of the flaky graphite.

The standard method of determining mechanical properties is the hardness test, in which indentations are made from the surface to the core. This method is destructive and time consuming. Because of this an easy nondestructive check-up of properties of the cast iron is highly desired. Various non-destructive evaluation techniques have been examined so far as an alternative method; alternating current potential drop [2], laser acoustic wave [3], ultrasonic back-scattering [4], eddy currents [5-7], photothermal radiometric radiometry [8]. 
Each technique gives indications of a good correlation between a measured physical parameter and hardness.

Magnetic measurements are also frequently used for characterization of changes in ferromagnetic materials, because magnetization processes are closely related to their microstructure. This makes the magnetic approach an obvious candidate for nondestructive testing, for detection and characterization of any defects in materials and in products made of such materials [see e.g. 9]. The well known Barkhausen noise effect can also be used for estimation of hardness in cast iron [10-13]. The so-called 3MAapproach (micromagnetic, multiple-parameter, microstructure, and stress analysis) was developed [14] in the last decade. This approach combines the information resulting from the performance of different micromagnetic techniques (magnetic Barkhausen noise, incremental permeability, harmonic analysis of the magnetic tangential field and eddy current testing used at 3 different frequencies). By using the 3MA- method a nondestructive hardness measurement is also possible [15].

A frequently and successfully used magnetic method is the measurement of hysteresis loops. This method is mostly based on detection of structural variations via the classical parameters of major hysteresis loops. Structural non-magnetic properties of ferromagnetic materials have been non-destructively tested using traditional hysteresis methods since long time with fair success. A number of techniques have been suggested, developed and currently used in industry, for a review see e.g. [16]. Hardening of steel is measured by detection of B-H loops as published is some recent works $[17,18]$. By applying this method, problems of non-destructive testing controlling the structure of casting products were analyzed, too. Coercive force, residual magnetization and saturation magnetization for white, gray, malleable and high-strength 
cast irons at different structure of metallic matrices were measured. It was found that measurement of the coercively sensitive magnetic parameter guarantees the quantitative control of hardness of casts without surface cleaning [19,20].

An alternative, more sensitive and more experimentally friendly approach to this topic was considered recently, based on magnetic minor loops measurement. The survey of this technique can be found in [21]. The method called Magnetic Adaptive Testing (MAT) was presented, which introduced general magnetic descriptors to diverse variations in non-magnetic properties of ferromagnetic materials, optimally adapted to the just investigated property and material. MAT was successfully applied for characterization of material degradation in different specimens and it seems to be an effective tool e.g. for replacement of the destructive hardness and/or ductile-brittle transition temperature measurements.

In our previous works [22-24] magnetic characteristic parameters of a system of minor loops, measured on a series of ductile cast iron samples, were analyzed, and their sensitivity was evaluated. The flat samples were magnetized by an attached yoke and sensitive parameters were obtained from the series of minor loops, without magnetic saturation of the samples, which characterize well the samplesôstructure. In a recent work [25] MAT was applied for three flake graphite cast iron materials with different chemical compositions and different matrix and flake graphite properties. Metallographic examination of the matrix and the graphite structures was performed and results of the non-destructive magnetic tests were compared with these data. A very good correlation was found between the magnetic descriptors and the graphite morphology. MAT was shown to be a useful tool for finding correlation between the chosen nondestructively measured magnetic parameters and the graphite morphology. 
Linear correlations with very small scatter of points were found between the optimally chosen MAT degradation functions and both the graphite length and the graphite area of the as-cast samples.

The purpose of the present work is to continue these measurements on three series of flake graphite cast iron samples, to investigate the influence of both graphite morphology structure and of matrices on mechanical and magnetic hardening, and to find correlation between nondestructively measured magnetic parameters and destructively determined Brinell hardness. We will also discuss the advantages of Magnetic Adaptive Testing compared with other existing nondestructive magnetic methods.

\section{Samples}

Three flake graphite cast iron materials with chemical compositions listed in Table 1 were prepared.

Table 1.

Chemical composition of the flake graphite cast iron samples (values in wt\%)

\begin{tabular}{|c|c|c|c|c|c|c|c|c|}
\hline \multirow{2}{*}{ Sample } & \multicolumn{7}{|c|}{ Chemical composition } & CE \\
\cline { 2 - 9 } & $\mathrm{C}$ & $\mathrm{Si}$ & $\mathrm{Mn}$ & $\mathrm{P}$ & $\mathrm{S}$ & $\mathrm{Cr}$ & $\mathrm{Ti}$ & $(\%)$ \\
\hline CE4.7 & 3.77 & 2.78 & 0.78 & 0.025 & 0.015 & 0.029 & 0.015 & 4.71 \\
\hline CE4.1 & 3.36 & 2.15 & 0.69 & 0.018 & 0.010 & 0.014 & 0.011 & 4.08 \\
\hline CE3.7 & 3.13 & 1.66 & 0.72 & 0.017 & 0.020 & 0.038 & 0.010 & 3.69 \\
\hline
\end{tabular}


Their carbon equivalent $(\mathrm{CE})$ values were defined by:

$$
C E=\operatorname{mass} \% \mathrm{C}+\frac{1}{3}(\operatorname{mass} \% \mathrm{Si}+\operatorname{mass} \% \mathrm{P})
$$

and were controlled to produce various graphite shapes and sizes. These metals were designated as CE4.7, CE4.1 and CE3.7 based on their targeted CE values. Pig iron (4.09\% C, 0.89\%Si, 0.07\%Mn, 0.019\%P, $0.012 \% \mathrm{~S}, 0.016 \% \mathrm{Cr}, 0.003 \% \mathrm{Ti})$, ferrosilicon (Fe-75\% Si), electrolytic iron and electrolytic manganese were used as raw materials and were melted using a high frequency induction melting furnace at $1743 \mathrm{~K}$. Ferrosilicon $(\mathrm{Fe}-75 \% \mathrm{Si})$ was also used as an inoculant. The melts were poured into moulds made by the $\mathrm{CO}_{2}$ gas process to produce the columnar bars with a length of $60 \mathrm{~mm}$ and a diameter of $46 \mathrm{~mm}$. Later each bar was cut into disks $10 \mathrm{~mm}$ thick. The disks were subjected to two kinds of heat treatments: annealing to obtain a ferrite based matrix and normalization to obtain a pearlite-based matrix. The disks intended for the heat treatments were kept in a furnace at $850^{\circ} \mathrm{C}$ for one hour and then either cooled in the furnace for the annealing or cooled in air for the normalization. We thus produced 3 ascast, 3 annealed and 3 normalized flake graphite cast iron materials with various matrices and graphite shapes as shown in Table 2.

After grinding the specimen surfaces, their Brinell hardness HB (HBW 10/3000) was measured and it is also listed in Table 2. These hardness values indicate that the furnace-cooling and air-cooling treatments were successful in producing the ferritic and pearlitic matrices, respectively.

Table 2.

Schedules of the heat treatment and the Brinell hardness (HBW) 


\begin{tabular}{|c|l|c|}
\hline Base material & \multicolumn{1}{|c|}{ Heat treatment } & HBW \\
\hline CE4.7 & as-cast & 100 \\
\hline CE4.7 & $850^{\circ} \mathrm{C} \times 1 \mathrm{~h}$, furnace-cooling & 89 \\
\hline CE4.7 & $850^{\circ} \mathrm{C} \times 1 \mathrm{~h}$, air-cooling & 130 \\
\hline CE4.1 & as-cast & 183 \\
\hline CE4.1 & $850^{\circ} \mathrm{C} \times 1 \mathrm{~h}$, furnace-cooling & 110 \\
\hline CE4.1 & $850^{\circ} \mathrm{C} \times 1 \mathrm{~h}$, air-cooling & 209 \\
\hline CE3.7 & as-cast & 207 \\
\hline CE3.7 & $850^{\circ} \mathrm{C} \times 1 \mathrm{~h}$, furnace-cooling & 130 \\
\hline CE3.7 & $850^{\circ} \mathrm{C} \times 1 \mathrm{~h}$, air-cooling & 221 \\
\hline
\end{tabular}

\section{Magnetic Adaptive Testing}

MAT investigates a complex set of minor hysteresis loops (from a minimum amplitude of the magnetizing field, with increasing amplitude by regular steps) for each sample of the measured series. It follows from the theory of Preisach model of hysteresis [26], that such a set of experimental data contains complex information on hysteresis of the measured material.

The essential difference between material testing by the traditional hysteresis- and by the MAT-approach is shown in Fig. 1 schematically. The left hand part of the figure represents the traditional measurement of the single major (saturation) hysteresis loop. The major loop is measured for each of the investigated samples and the material degradation can be described through variation of values of any of the few major loop parameters, e.g. $H_{C}, B_{R}, \ldots$ as functions of an independent degradation variable, $\varepsilon$. The 
right hand part of the figure depicts schematically volume of the measured data for MAT. The large family of minor hysteresis loops is measured for each of the investigated samples and the material degradation can be then described through variation of values of any of the point (and/or slope) on any of the minor loops, i.e. $B\left(F_{i}, A_{j}\right)$ (and/or $\left.\mu\left(F_{i}, A_{j}\right)\right)$, as functions of any independent degradation variable, $\varepsilon$. . In the present case the Brinell hardness is the independent parameter, i.e. $H B W$ values will be used later as $\varepsilon$.

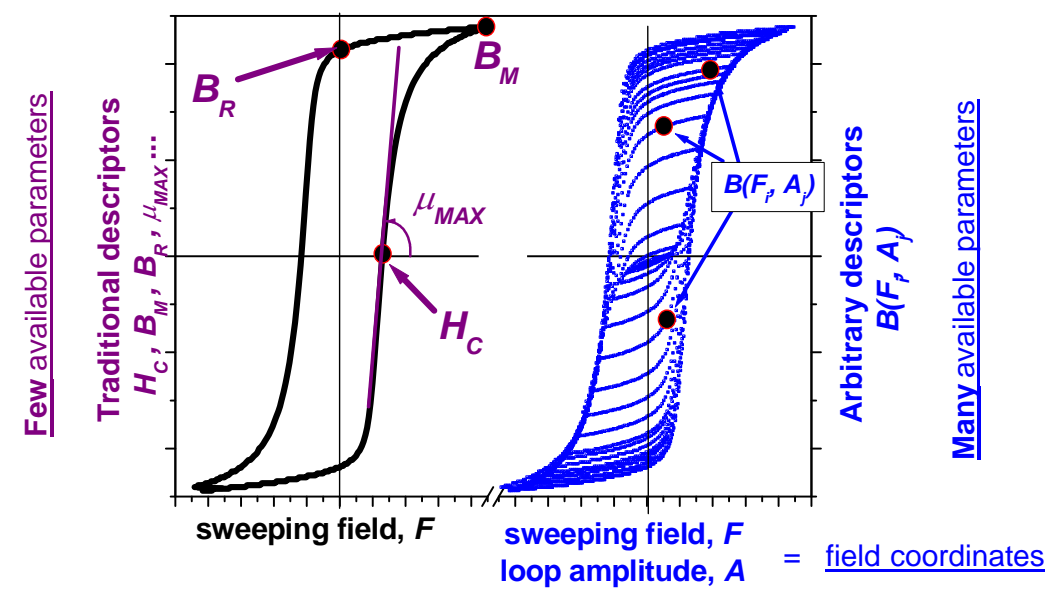

Fig. 1. Schematic comparison of the traditional magnetic hysteresis testing (left) and Magnetic Adaptive Testing (right). The traditional testing uses only a few parameters of the major loop for description of the material. Magnetic Adaptive Testing has the choice to pick up the best from many available parameters indexed by the field coordinates.

The method of Magnetic Adaptive Testing utilizes systematic measurement of large families of minor hysteresis loops, from minimum amplitudes up to possibly the maximum (major) ones on degraded ferromagnetic samples/objects. From the large volume of the recorded data, those are applied for evaluation of the degradation, which reflect the material degradation in the most sensitive or otherwise the most convenient 
way. Such $\ddot{i}$ best adapted for the investigated case $\ddot{i}$ data are used as the MATparameter(s) and its / their dependence on an independent variable accompanying the inspected degradation is referred to as the MAT degradation function(s).

The magnetic induction method appears to be the easiest way of the systematic measurement for MAT. A specially designed Permeameter [27] with a magnetizing yoke was applied for measurement of families of minor loops of the magnetic circuit differential permeability. The measurement of the hysteresis loops is performed by a magnetizing yoke, which is placed on the flat surface of the sample. A C-shaped laminated Fe-Si transformer core was used. The block-scheme of the device and the sketch of the yoke can be seen in Fig. 2a. The driving coil wound on the yoke produces triangular variations of the applied magnetic field with stepwise increasing amplitudes and with a fixed slope magnitude in all the triangles (see Fig. 2b).

The signal coil picks-up the induced voltage proportional to differential permeability of the sample. This triangular variation of the magnetizing field with time, $t$, and a voltage signal, $U$, is induced in the pick-up coil for each $k^{\text {th }}$ sample:

$U\left(\mathrm{~d} F / \mathrm{d} t, F, A_{j}, \varepsilon_{k}\right)=K^{*} \partial B\left(\mathrm{~d} F / \mathrm{d} t, F, A_{j}, \varepsilon_{k}\right) / \partial t=K^{*} \mu\left(\mathrm{d} F / \mathrm{d} t, F, A_{j}, \varepsilon_{k}\right)^{*} \mathrm{~d} F / \mathrm{d} t$,

where $K$ is a constant determined by geometry of the sample and by the experimental arrangement. As long as $F=F(t)$ sweeps linearly with time $\ddot{i}$ i.e. $|\mathrm{d} F / \mathrm{d} t|$ is (the same) constant for measurement at each of the samples, Eq.(1) states, that the measured signal is simply proportional to the differential permeability, $\mu$, of the measured magnetic circuit, as it varies with the applied field, $F$, within each minor loop amplitude, $A_{j}$, for each $k^{\text {th }}$ measured sample. If we wish to get correct results without influence of any 
previous remanence, it is evident that each sample has to be thoroughly demagnetized before it is measured.

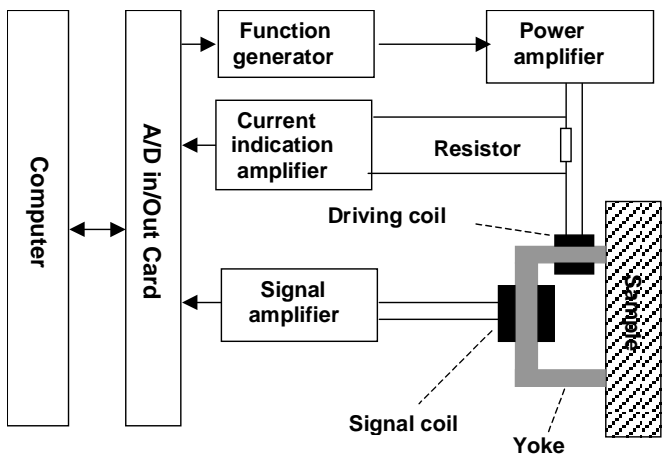

a)

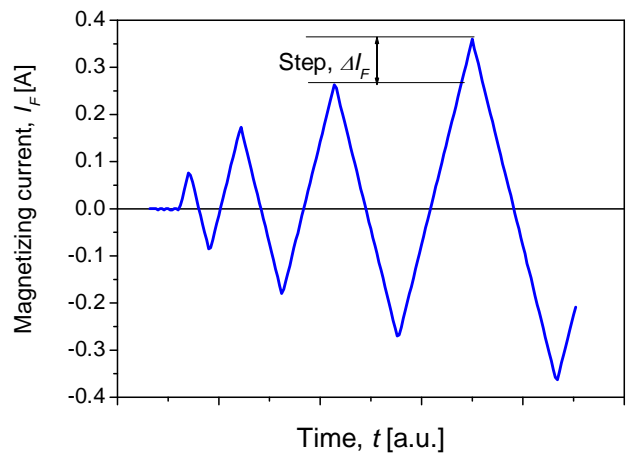

b)

Fig. 2. a) Block-scheme of the Permeameter and sketch of the yoke, b) Triangular variation of the magnetizing current with time.

The Permeameter works under control of a notebook PC, which sends the steering information to the function generator and collects the measured data. An input/output data acquisition card accomplishes the measurement. The computer registers actually two data files for each measured family of the minor $\mu$-shaped loops. The first one contains detailed information about all the pre-selected parameters of the demagnetization and of the measurement. The other file holds the course of the voltage signal, $U$, induced in the pick-up coil as a function of time, $t$, and of the magnetizing current, $I_{F}$, and/or field, $F$. As an illustration, Fig. 3 presents the three families of permeability loops, measured on the three as cast samples (CE3.7, CE4.1, CE4.7). Evidently it is a lot of data and our task is to compare them and to find the most suitable ones for characterizing the changes between samples. 


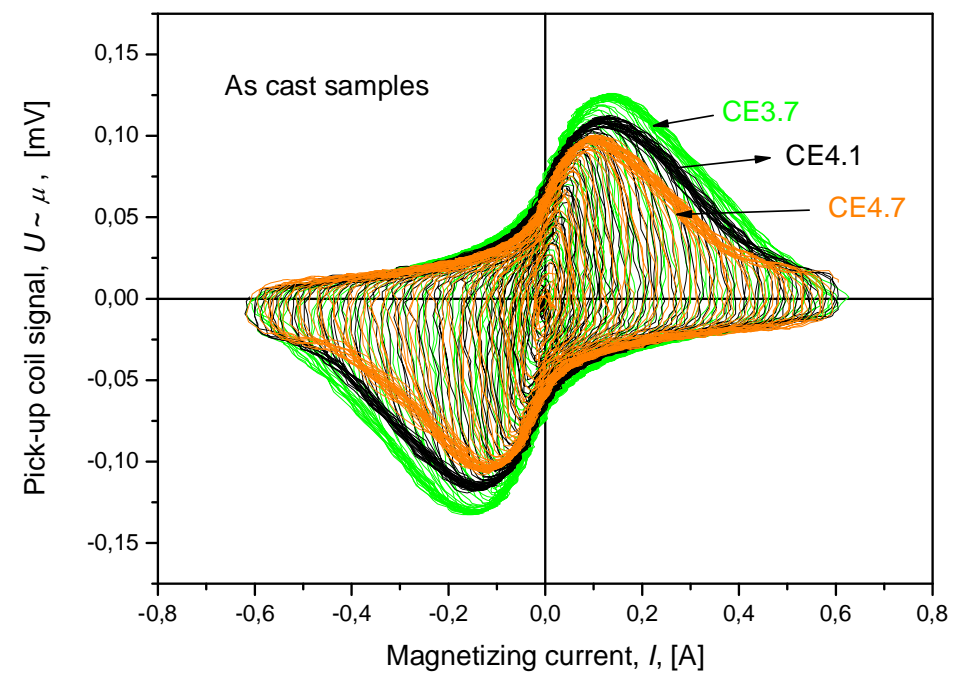

Fig. 3. Examples of families of the $\mu$-shaped loops vs. magnetizing current, $I_{F}$, measured on the three as cast samples. The positive and negative parts of the signal correspond to the increasing and decreasing parts of the triangular waveform of the current, respectively.

Instead of keeping the signal and the magnetizing field in shapes of continuous time-dependent functions, it is practical to interpolate the family of data for each $\varepsilon_{k^{-}}$ sample into a discrete square $(i, j)$-matrix, $U\left(F_{i}, A_{j}, \varepsilon_{k}\right)$, with a suitably chosen step, $\Delta A=\Delta F$. (Because $\mathrm{d} F / \mathrm{d} t$ is a constant, identical for all measurements within one experiment, it is not necessary to write it explicitly as a variable of $U$.) MAT is a relative method (practically all the nondestructive methods are relative), and the most suitable information about degradation of the investigated material can be contained in variation of any element, of such matrices as a function of $\varepsilon$, relative with respect to the corresponding element of the reference matrix, $U\left(F_{i}, A_{j}, \varepsilon_{0}\right)$. So that we shall divide all $U\left(F_{i}, A_{j}, \varepsilon_{k}\right)$ elements by the corresponding elements $U\left(F_{i}, A_{j}, \varepsilon_{0}\right)$ of the reference sample 
matrix and obtain normalized elements of matrices of relative differential permeability $\mu\left(F_{i}, A_{j}, \varepsilon_{k}\right)=U\left(F_{i}, A_{j}, \varepsilon_{k}\right) / U\left(F_{i}, A_{j}, \varepsilon_{0}\right)$, and their proper sequences

$$
\mu\left(F_{i}, A_{j}, \varepsilon\right)=U\left(F_{i}, A_{j}, \varepsilon\right) / U\left(F_{i}, A_{j}, \varepsilon_{0}\right)
$$

as normalized $\mu$-degradation functions of the inspected material.

In some cases it turns out, that degradation functions of reciprocal values, such as $1 / \mu$-degradation functions are more convenient than the direct ones. Application of the reciprocal degradation functions proves effective especially in situations when $\ddot{i}$ with the increasing parameter $\varepsilon \ddot{i}$ the direct degradation functions approach kind of a ñsaturationò. Number of the degradation functions obtained from the MAT measurement depends on magnitude of the maximum minor loop amplitude, $A_{j}$, up to which the measurement is done, and on choice of the step value $\Delta A=\Delta F$ which is used for computation of the interpolated data matrices.

Once the degradation functions are computed, the next task is to find the optimum degradation function(s) for the most sensitive and enough robust description of the investigated material degradation. A 3D-plot of sensitivity of the degradation functions can substantially help to choose the optimum one(s). For illustration, map of relative sensitivity of the $1 / \mu_{i j}(H B W)$-degradation functions in the case of the as cast samples is shown in Fig. 9. Slope of the linear regression of each degradation function is here defined as the function sensitivity. Thus the sensitivity map is a 3D-graph of these slope values plotted against the degradation functions field coordinates $\left(F_{i}, A_{j}\right)$. As it follows from the presented sensitivity maps, in Fig. 9 the most sensitive $1 / \mu$ - 
degradation functions are those with field coordinates around $\left(F_{i}=-700 \mathrm{~mA}, A_{j}=725 \mathrm{~mA}\right)$. The most sensitive $1 / \mu$-degradation functions are plotted in Fig. 6.

Size of the yoke was chosen to fit geometry of the samples: cross-section $S=10 \times 5 \mathrm{~mm}^{2}$, the total outside length $18 \mathrm{~mm}$, and the total outside height of the bow $22 \mathrm{~mm}$. The magnetizing coil was wound on the bow of the yoke, with $N=200$ turns and the pick-up (or signal) coil was wound on one of the yoke legs with $n=75$ turns.

\section{Results}

The metallographic examination of the matrix and graphite structures were done according to ISO 945 [25]. Microphotographs of the three materials in their as-cast condition revealed that the graphite flakes of CE4.7 are relatively long, they are uniformly and isotropically distributed, and are thus categorized as type-B flakes defined by ISO 945. CE4.1 has smaller graphite flakes than CE4.7 and they are categorized as type-A flakes. In CE3.7 very small eutectic graphite flakes were found to be distributed in the dendrite and they are categorized as type-D and type-E flakes. Microphotographs of the samples after etching with 3\% Nital indicated that CE4.7 had a pearlite-ferrite matrix, CE3.7 had a completely pearlitic matrix, and CE4.1 mainly had a pearlitic matrix and a small amount of ferrite surrounded the graphite flakes. The area fraction and the average length of the graphite flakes were evaluated using an image processing software. The area fraction of graphite was evaluated using microphotograph binary images of 5 sample regions at the same magnification. The length of graphite is defined as the average diameter of the minimum circle circumscribing each graphite flake larger than $5 \mu \mathrm{m}$. The area fraction of graphite for CE4.7, CE4.1 and CE3.7 is 
$17.8,12.6$ and $10.0 \%$, respectively. The length of the graphite flakes for CE4.7, CE4.1 and CE3.7 is 67, 39 and $28 \mu \mathrm{m}$, respectively. For details see [25].

MAT degradation functions of all the investigated samples were evaluated and those, optimized for description of the studied dependences, were considered as functions of Brinell hardness. Optimization means that those $\mu_{i j}(H B W)$-degradation functions were chosen from the big data pool, which were the most sensitive with respect to the change of the independent parameter, and at the same time they were highly repeatable, and in such a way the most reliable.

The results for the three different materials are given in Fig. 4. Here each graph within the same figure represents one composition (CE4.7, CE4.1 and CE3.7) and the type of cooling condition (as-cast, furnace-cooling, air-cooling) is also indicated. In every case the MAT parameters are standardized by the corresponding value of the sample within the same series, which has the lowest $H B W$. The optimum of MAT descriptors in this case was the $1 / \mu_{i j}(H B W)$-degradation function, with $\left(F_{i}=0, A_{j}=600\right.$ $\mathrm{mA}$ ) values.

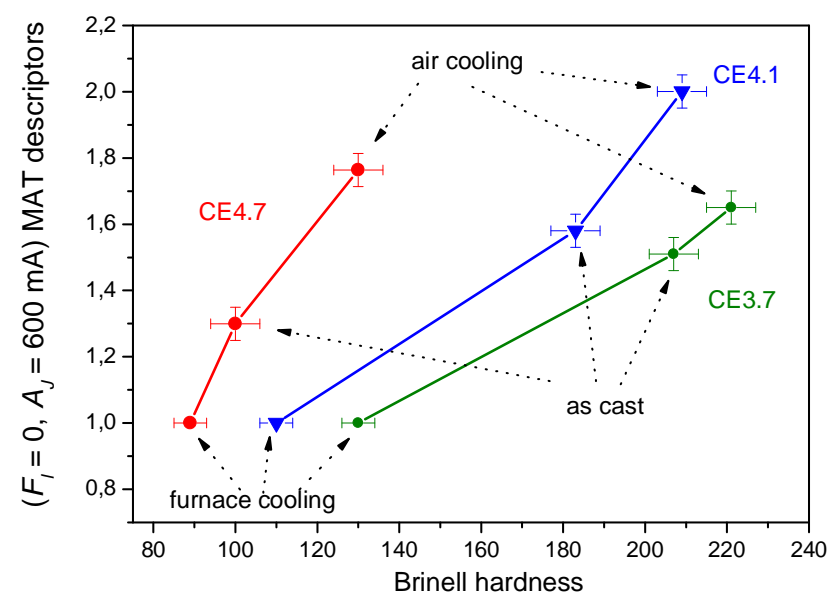


Fig. 4. The optimally chosen $1 / \mu$ MAT degradation functions of the three sample series vs. Brinell hardness.

The corresponding sensitivity map is shown in Fig. 5. This shows the result of the measurement, where MAT parameters were measured as a function of hardness on the CE3.7 sample series. It is seen very well that the most sensitive area is around the $\left(F_{i}=0, A_{j}=600 \mathrm{~mA}\right)$ values (the red area in the figure, indicated also by the crossing lines). It can also be observed that the sensitive area is rather large, which ensures the good reproducibility of the measurement. Very similar sensitivity maps were obtained if the measurements were performed on the CE4.1 and on the CE4.7 sample series (not shown here). The $1 / \mu_{i j}(H B W)$-degradation function $\left(F_{i}=0, A_{j}=600 \mathrm{~mA}\right)$ was chosen as the optimal MAT descriptor in Fig. 4 for all the samples, in consistency with these results.

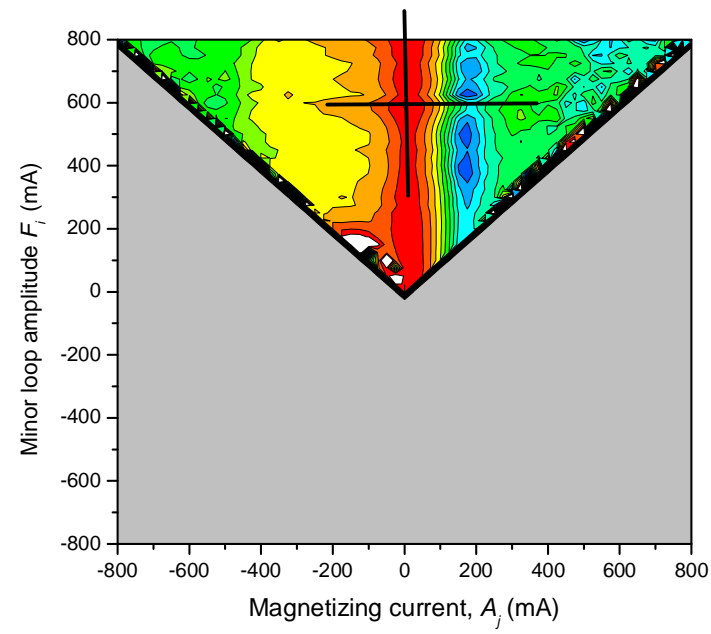

Fig. 5. Map of relative sensitivity of the $1 / \mu_{i j}(H B W)$-degradation functions in the case of CE3.7 sample. The crossing lines show the point, from where descriptors of Fig. 4 were $\operatorname{taken}\left(F_{i}=0, A_{j}=600 \mathrm{~mA}\right)$. 
The three graphs in Fig. 4 show the connection between the magnetic parameters and the Brinell hardness within the same series (the same chemical composition) of the samples. Different heat treatments result in different values of hardness.

However, the hardness (and simultaneously the magnetic parameters) are also modified depending on graphite morphology, if the samples are prepared by the same procedure (as-cast, furnace-cooling, air-cooling). The best MAT degradation functions vs. Brinell hardness, optimized for the as cast samples, are shown in Fig. 6. The same is shown in Fig. 7 and Fig. 8 for the furnace-cooled and for the air-cooled samples, respectively. In all these cases (Figs. 6, 7 and 8) the optimum of MAT descriptor was the $1 / \mu_{i j}(H B W)$-degradation function, with $\left(F_{i}=-700 \mathrm{~mA}, A_{j}=725 \mathrm{~mA}\right)$ values.

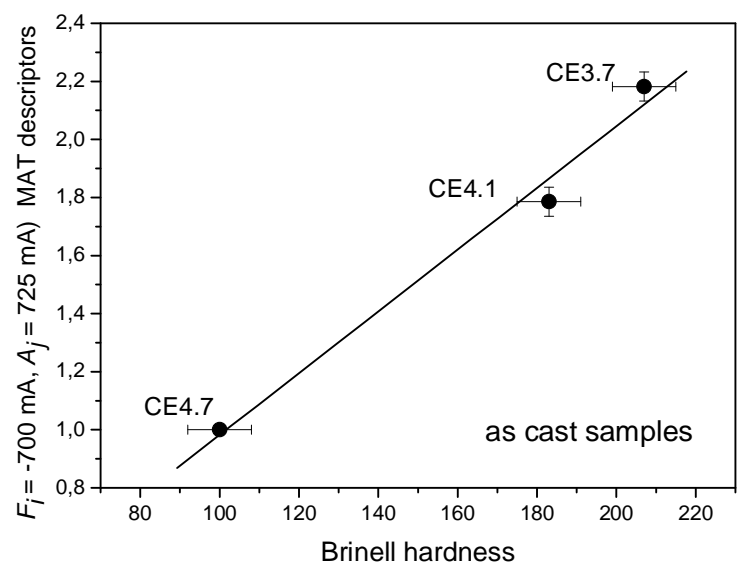

Fig. 6. The optimally chosen $1 / \mu$ MAT degradation function for the as-cast samples vs. Brinell hardness. 


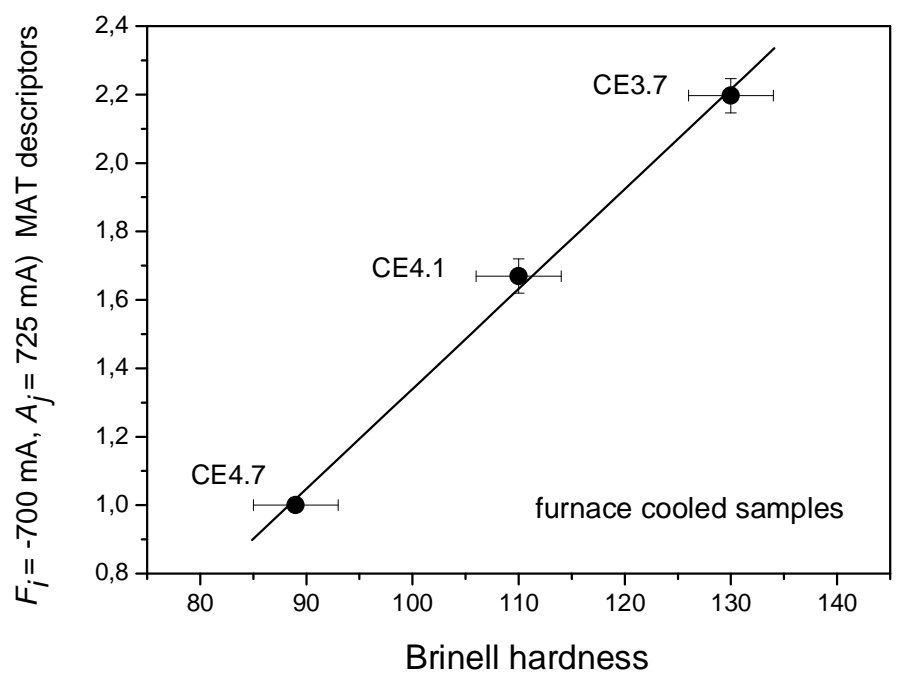

Fig. 7. The optimally chosen $1 / \mu$ MAT degradation function for the furnace-cooled samples vs. Brinell hardness.

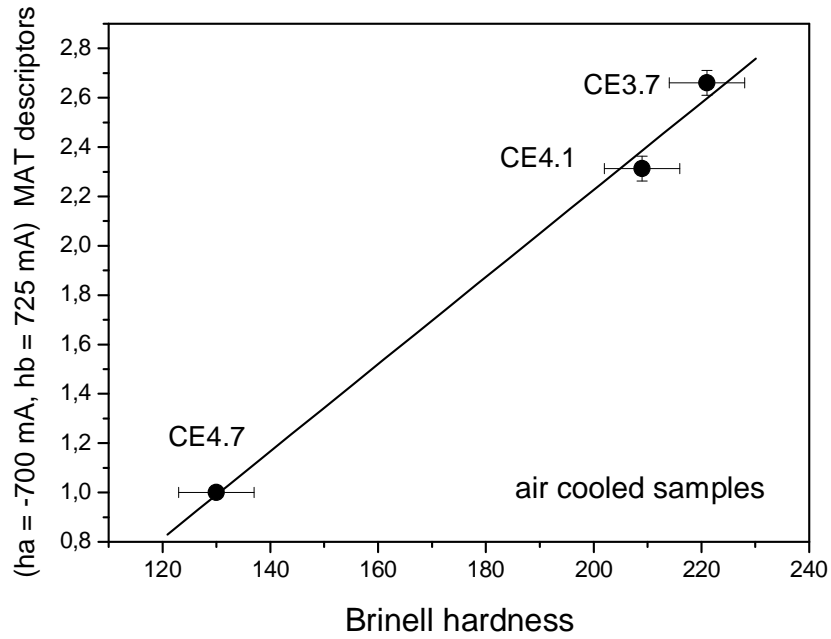

Fig. 8. The optimally chosen $1 / \mu$ MAT degradation function for the air-cooled samples vs. Brinell hardness.

The corresponding sensitivity map of Fig. 6 is shown in Fig. 9. This sensitivity map shows the result of the measurement, where MAT parameters were measured as a 
function of hardness on the three as cast samples. The most sensitive area is around $\left(F_{i}\right.$ $=-700 \mathrm{~mA}, A_{j}=725 \mathrm{~mA}$ ) values (the red area in the figure, indicated also by the crossing lines). It can also be observed that the sensitive area is large enough, which ensures the good reproducibility of the measurement. Very similar sensitivity maps were obtained if the measurements were performed on the air cooled and on the furnace cooled sample series. It means that in Figs. 6, 7 and 8 the optimally chosen MAT descriptor is the $1 / \mu_{i j}(H B W)$-degradation function $\left(F_{i}=-700 \mathrm{~mA}, A_{j}=725 \mathrm{~mA}\right)$ for all the three sample series.

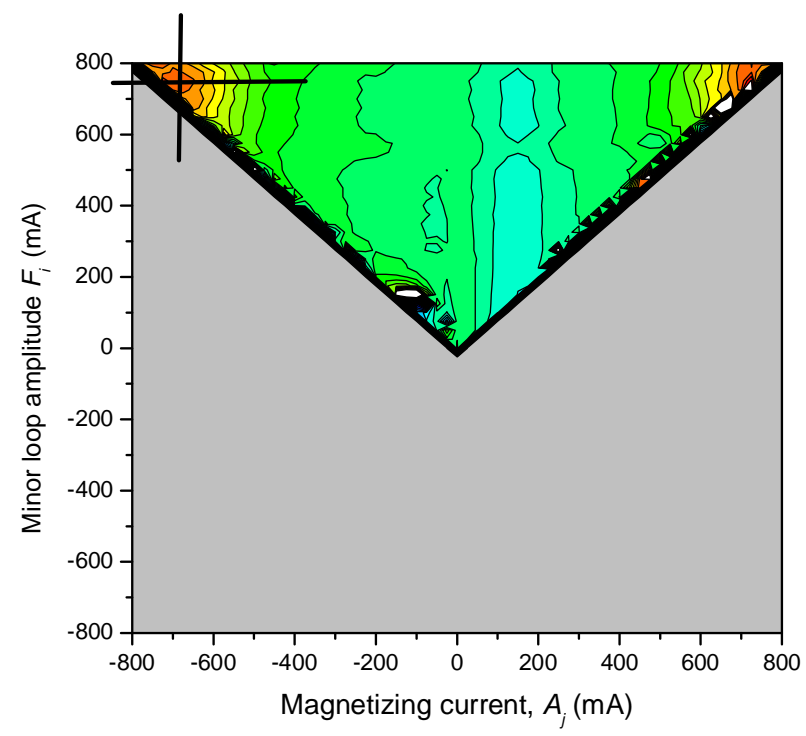

Fig. 9. Map of relative sensitivity of the $1 / \mu_{i j}(H B W)$-degradation functions in the case of the as cast samples. The crossing lines show the point, from where descriptors of Fig. 6 were taken $\left(F_{i}=-700 \mathrm{~mA}, A_{j}=725 \mathrm{~mA}\right)$.

\section{Discussion}

The samples are magnetized during the measurement by a magnetizing yoke, which is placed on the flat surface of the sample. This experimental arrangement means 
an open magnetic circuit, because some magnetic flux is always scattered at the air gap between the yoke and the sample. To get reliable MAT-data, quality of the surface must not vary from sample to sample and conditions of the measurement must be kept constant within the each series of experiments. The exact value of the magnetic field inside the sample is not known/measured in the used experimental arrangement. Because of this, instead of the magnetic field (given in $\mathrm{A} / \mathrm{m}$ ), the value of the magnetizing current (given in $\mathrm{mA}$ ) is used as $F_{i j}$ and $A_{i j}$ when the $\mu_{i j} \equiv \mu\left(F_{i j}, A_{i j}\right)$ matrix elements are given.

MAT parameters also depend on the microstructure state (graphite morphology), because graphite morphology determines the pearlite-ferrite ratio of the material. This is also very well reflected by magnetic measurements: very good correlation was found between MAT parameters and graphite morphology. The correlation between MAT parameters and both graphite length and graphite area was shown and discussed in Ref. [25].

As it is seen in the figures, closely linear correlation was found between the optimized MAT degradation functions and Brinell hardness in all the investigated cases. It was applicable if the influence of different cooling conditions was investigated within the same series of the samples (the same chemical composition, which leads to different graphite structure), and also, if the influence of chemical composition was studied for identically heat-treated samples. This confirms the fact that magnetic hardening follows the mechanical hardening very well and that Magnetic Adaptive Testing is a powerful tool for the nondestructive determination of this hardening.

There are several data in the literature about correlation between nondestructively measured magnetic parameters (characteristics of major hysteresis loop, Barkhausen 
noise measurements, etc.). In all cases a linear correlation was found between the magnetic parameters and hardness $[7,11,12,13,19,28]$. This coincidence is rather promising from that point of view, that magnetic measurements in general ï regardless on the actual type of investigation $\ddot{i}$ reflect the changes in mechanical hardening and in such a way they can replace the destructive and time consuming present standard way of inspection. The question is, which magnetic measurement can be most successfully applied. Based on our experience, we believe that MAT can be a suitable candidate for future practical application. There are two important arguments, which support this assumption. Our experience with previous MAT measurements, performed on different samples showed that if different types of magnetic measurements (major hysteresis loop, Barkhausen noise measurement) are applied on the same series of deformed samples, MAT is more sensitive that other methods [21]. The other one is, that during the MAT measurement there is no need for magnetic saturation of the investigated sample, which is a big advantage. In practical applications, where big and complicated shape samples should be measured, magnetic saturation is almost impossible.

We had alltogether nine samples, e.g. three series with three different samples within each series. This means that the graphs contain only three measured points. From the point of view of statistics this seems to be a rather low number, but considering the large difference between the measured values, both in MAT parameters and hardness, the low error of the measured points and the low scatter of points around the hypothetical linear correlation, we believe that the results are reliable enough to prove the correlation between magnetic parameters and independent parameter.

The above presented results reflect another important feature of Magnetic Adaptive Testing, too. Namely its multiparametric character. Made one single 
measurement on the investigated sample a big data pool is generated. The method of Magnetic Adaptive Testing looks for those magnetic descriptors of the varied structural properties, which are best adapted to the investigated property and to the investigated material. It is seen on the above presented figures that different MAT descriptors were used for characterization of material. If any series of samples with the same chemical composition but with different thermal processing was considered, the $1 / \mu_{i j}(H B W)$ degradation function with parameters $\left(F_{i}=0, A_{j}=600 \mathrm{~mA}\right)$ gave good result. Another $1 / \mu_{i j}(H B W)$-degradation function with parameters $\left(F_{i}=-700 \mathrm{~mA}, A_{j}=725 \mathrm{~mA}\right)$ reflected the hardness if different chemical composition samples with the same thermal processing were considered. It is emphasized again that all of these degradation functions were evaluated from one single measurement.

It is important to emphasize that MAT is a relative measurement: in all cases we compare the parameters of measured samples with the parameters of the reference (virgin) sample. For the successful application of the MAT method, first it is necessary to make comparative, traditional, destructive measurements on a series of samples, for ñteachingò the MAT. This teaching procedure determines the optimum degradation function/s, and the method of Magnetic Adaptive Testing is best adapted to the investigated task in this way. Then, this/these chosen optimum degradation function(s) will serve as sensitive calibration curve(s) for practical measurements on unknown samples (of the same kind) to be investigated. Obviously different measuring conditions result in different relative sensitivity of the calculated descriptors. Because of this identical experimental conditions should be rigorously kept during measurement of the tested objects, as they were applied during evaluation of the reference samples series. If we do this, the reproducibility of the MAT parameters is excellent even replacing the 
circuit after longer use in practice, and we detect only the material modification because of the possible wear. The validity of this statement was tested by several control measurements.

\section{Conclusions}

The method Magnetic Adaptive Testing, which is based on nondestructive, systematic measurement of minor magnetic hysteresis loops was applied for three flake graphite cast iron series having different chemical compositions and different heat treatments within each series. MAT was shown to be a useful tool for finding correlation between the nondestructively measured magnetic parameters and Brinell hardness. Linear correlations with very small scatter of points were found between the optimally chosen MAT degradation functions and the actual value of the Brinell hardness, regardless if the chemical composition or the way of heat treatment was considered. Also, referring to our previous work in this subject, good correlation was found between MAT parameters and graphite morphology of as cast samples.

As a consequence, Magnetic Adaptive Testing proved to be an experimentally friendly and sensitive method for nondestructive tests of the cast iron structure.

\section{Acknowledgments}

This work was partly supported by the JSPS Core-to-Core Program, ĂAdvanced Research Networksò (International research core on smart layered materials and structures for energy saving), by Hungarian Scientific Research Fund (project K 111662) and by the FY2013 Researcher Exchange Program between the Japan Society for Promotion of Science and Hungarian Academy of Sciences. One of the coauthors (I.T.) appreciates financial support by the project TA02011179 of the Technical 
Agency of the Czech Republic.

\section{References}

[1] Walton CF, Opar TJ(Eds.), Iron casting handbook, Iron Casting Society, Inc., New York, 1981.

[2] Bowler JR, Huang Y, Sun H, Brown J, Bowler N, Alternating current potentialdrop measurement of the depth of case hardening in steel rods, Measurement Science and Technology 19 (2008) 075204.

[3] Schneider D, Hofmann R, Schwarz T, Grosser T, Hensel E, Evaluating surface hardened steels by laser-acoustics Surface and Coatings Technology 206 (2012) 2079.

[4] Willems H, Review of Progress in Quantitative Nondestructive Evaluation 10B (1991) 1707.

[5] Uchimoto T, Takagi T, Konoplyuk S, Abe T, Huang H, Kurosawa M, Eddy current evaluation of cast iron for material characterization, J. Magn. Magn. Mater., 258259 (2003) 493.

[6] Konoplyuk S, Abe T, Uchimoto T, Takagi T, Kurosawa M, Characterization of ductile cast iron by eddy current method, NDT\&E International, 38 (2005) 623

[7] Feiste KL, Fetter Marques P, Reichert Ch, Reimche W, Stegemann D, Rebello AJM, Krüger ES, Characterization of Nodular Cast Iron Properties by Harmonic Analysis of Eddy Current Signals, http://ndt.net/article/ecndt98/nuclear/245/245.htm

[8] Wang C, Mandelis A, Case depth determination in heat-treated industrial steel products using photothermal radiometric interferometric phase minima, NDT\&E International 40 (2007) 158. 
[9] Jiles DC, Magnetic methods in nondestructive testing, Buschow KHJ , Ed., Encyclopedia of Materials Science and Technology, Elsevier Press, Oxford, p.6021, 2001

[10] Santa-aho S, Vippola M, Sorsa A, Leiviskä K, Lindgren M, Lepistö T, Utilization of Barkhausen noise magnetizing sweeps for case-depth detection from hardened steel, NDTE\&E International, 52 (2012) 95-102

[11] Helmersson PL, Thibblin A, Method for handling a cast iron component based on estimating hardness by magnetic Barkhausen noise, USPTO Application \#:\#20080238418 - Class: 324234 (USPTO) - 10/02/08 - Class 324

[12] Pirfo Barroso S, Horváth M, Horváth Á, Magnetic measurements for evaluation of radiation damage on nuclear reactor materials, Nuclear Engineering and Desing 240 (2010) 722-725

[13] Luo XY, Zhang Y, Wang ZJ, Zhang YS, Non-destructive Testing Device for Hot Forming High Strength Steel Parts Based on Barkhausen Noise, Applied Materials and Technologies for Modern Manufacturing, PTS 1-4, 2013, Volume: 423-426, pp. $2555-2558$

[14] Dobmann G, (2011). Non-Destructive Testing for Ageing Management of Nuclear Power Components, Nuclear Power - Control, Reliability and Human Factors, Dr. Pavel Tsvetkov (Ed.), ISBN: 978-953-307-599-0, InTech, DOI: 10.5772/17581. Available from: http://www.intechopen.com/books/nuclearpower-control-reliability-and-human-factors/non-destructive-testing-for-ageingmanagement-of-nuclear-power-components 
[15] Altpeter I, Becker R, Dobmann G, Kern R, Theiner A, Yashan A, Robust solutions of inverse problems in electromagnetic non-destructive evaluation, Inverse Problems, 18 (2002) 1907-1921

[16] Blitz J, Electrical and magnetic methods of nondestructive testing, Bristol, Adam Hilger IOP Publishing, Ltd., 1991.

[17] Zhang C, Bowler N, Lo C, Magnetic characterization of surface-hardened steel, J. Magn. Magn. Mater, 321 (2009) 3878ї 3887

[18] Kobayashi S,Takahashi S, Kamada Y, Evaluation of case-depth in inductionhardened steels: Magnetic hysteresis measurements and hardness-depth profiling by differential permeability analysis, J. Magn. Magn. Mater., 343 (2013) 112-118

[19] Sandomirsky SG, Magnetic testing of structure of the cast-iron products: possibilities and results, www.ndt.net/article/ecndt2010/reports/1_01_21.pdf

[20] Sandomirsky SG, Tsukerman VL, Pisarenko LZ, Possibilities and results of hardness control of cast iron castings by magnetic method after polar

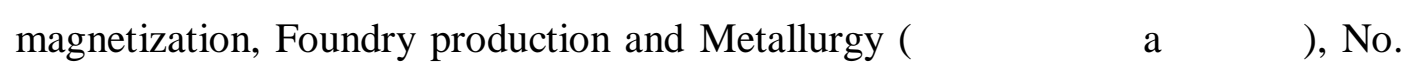
3 (2007) pp. 106-110

[21] Tomáǵ I, Vértesy G, Magnetic Adaptive Testing, in Nondestructive Testing Methods and New Applications, M.Omar (Ed.), ISBN: 978-953-51-0108-6, (2012), InTech: http://www.intechopen.com/articles/show/title/magnetic-adaptivetesting.

[22] Vértesy G, Uchimoto T, Takagi T, Tomáǵ I, Stupakov O, Meszaros I, Pavo J, Minor hysteresis loops measurements for characterization of cast iron, Physica B, 372 (2006), pp. 156-159 
[23] Vértesy G, Uchimoto T, Tomág I, Takagi T, Nondestructive characterization of ductile cast iron by Magnetic Adaptive Testing, J.Magn.Magn.Mater. 322 (2010) 3117-3121.

[24] TomáǵI, Skrbek B, Uchimoto T, Kadlecová J, Stupakov O, Perevertov O, Doḷ ekal J, Application of Magnetic Adaptive Testing to Cast Iron, Acta Metallurgica Slovaca, 13 (2007) pp.129 ï 132.

[25] Vértesy G, Uchimoto T, Takagi T, Tomáġ I, Flake graphite cast iron investigated by a magnetic method, IEEE Trans. Magn, Vol. 50. No. 4, April 2014, 6200404

[26] Mayergoyz ID, Mathematical models of hysteresis, Springer-Verlag, New York, 1991

[27] Tomáġ I, Perevertov O, Permeameter for Preisach approach to materials testing, JSAEM Studies in Applied Electromagnetics and Mechanics 9, Ed. Takagi T, Ueasaka M, IOS Press, Amsterdam, p. 5., 2001.

[28] Fillion G, Lord M, Bussiere JF, Inference of hardness from magnetic measurement on pearlitic steels, Review of Progress in Quantitative Nondestructive Evaluation, Vol. 9, Eds. Thomson DO, Climenti DE, Plenum Press, New York, 1990 


\section{Figure captions}

Fig. 1: Schematic comparison of the traditional magnetic hysteresis testing (left) and Magnetic Adaptive Testing (right). The traditional testing uses only a few parameters of the major loop for description of the material. Magnetic Adaptive Testing has the choice to pick up the best from many available parameters indexed by the field coordinates.

Fig. 2: a) Block-scheme of the Permeameter and sketch of the yoke, b) Triangular variation of the magnetizing current with time.

Fig. 3: Examples of families of the $\mu$-shaped loops vs. magnetizing current, $I_{F}$, measured on the three as cast samples. The positive and negative parts of the signal correspond to the increasing and decreasing parts of the triangular waveform of the current, respectively.

Fig. 4: The optimally chosen $1 / \mu$ MAT degradation functions of the three sample series vs. Brinell hardness.

Fig. 5: Map of relative sensitivity of the $1 / \mu_{i j}(H B W)$-degradation functions in the case of CE3.7 sample. The crossing lines show the point, from where descriptors of Fig. 4 were taken $\left(F_{i}=0, A_{j}=600 \mathrm{~mA}\right)$.

Fig. 6: The optimally chosen $1 / \mu$ MAT degradation function for the as-cast samples vs. Brinell hardness.

Fig. 7: The optimally chosen $1 / \mu$ MAT degradation function for the furnace-cooled samples vs. Brinell hardness. 
Fig. 8: The optimally chosen $1 / \mu$ MAT degradation function for the air-cooled samples vs. Brinell hardness.

Fig. 9: Map of relative sensitivity of the $1 / \mu_{i j}(H B W)$-degradation functions in the case of the as cast samples. The crossing lines show the point, from where descriptors of Fig. 6 were taken $\left(F_{i}=-700 \mathrm{~mA}, A_{j}=725 \mathrm{~mA}\right)$. 